\title{
Long-Term Memory Formation in Drosophila Requires Training-Dependent Glial Transcription
}

\author{
Motomi Matsuno, ${ }^{1}$ Junjiro Horiuchi, ${ }^{1}$ Yoshihiro Yuasa, ${ }^{2,3}$ Kyoko Ofusa, ${ }^{1}$ Tomoyuki Miyashita, ${ }^{1}$ Tomoko Masuda, ${ }^{1}$ \\ and Minoru Saitoe ${ }^{1}$ \\ ${ }^{1}$ Department of Sensory and Motor Systems, Tokyo Metropolitan Institute of Medical Science, Tokyo 156-0057, Japan, ${ }^{2}$ Institut de Génétique et de Biologie \\ Moléculaire et Cellulaire (IGMBC), 67404 Illkirch-Graffenstaden, France, and ${ }^{3}$ Department of Developmental Genetics, National Institute of Genetics, \\ Shizuoka 411-8540, Japan
}

Long-term memory (LTM) formation requires de novo gene expression in neurons, and subsequent structural and functional modification of synapses. However, the importance of gene expression in glia during this process has not been well studied. In this report, we characterize a cell adhesion molecule, Klingon (Klg), which is required for LTM formation in Drosophila. We found that Klg localizes to the juncture between neurons and glia, and expression in both cell types is required for LTM. We further found that expression of a glial gene, repo, is reduced in $k \mathrm{lg}$ mutants and knockdown lines. repo expression is required for LTM, and expression increases upon LTM induction. In addition, increasing repo expression in glia is sufficient to restore LTM in $\mathrm{klg}$ knockdown lines. These data indicate that neuronal activity enhances Klg-mediated neuron-glia interactions, causing an increase in glial expression of repo. Repo is a homeodomain transcription factor, suggesting that further downstream glial gene expression is also required for LTM.

Key words: Drosophila; glial transcription; long-term memory; repo

\section{Introduction}

Glia play critical roles in synaptic plasticity and memory formation by regulating neurotransmitter and ion concentrations in extracellular spaces, supplying energy sources and nutrients to neurons, and mediating activity-dependent morphological changes in synapses (Suzuki et al., 2011; Pannasch and Rouach, 2013; Zwarts et al., 2014). They also respond acutely to neuronal damage by inducing morphological changes required for axonal engulfment and clearance (MacDonald et al., 2006; Doherty et al., 2009). These functions demonstrate that glia can sense, and respond to, changes in neuronal states. However, while some glial proteins have been shown to be regulated by neuronal activity (Steward et al., 1991; Comas et al., 2004; Muthukumar et al., 2014), changes in activity of glia-specific transcription factors,

Received Sept. 17, 2014; revised Feb. 3, 2015; accepted Feb. 28, 2015.

Author contributions: M.M. and M.S. designed research; M.M., J.H., Y.Y., K.O., T. Miyashita, and T. Masuda performed research; Y.Y. contributed unpublished reagents/analytic tools; M.M., J.H., and M.S. analyzed data;M.M., J.H., and M.S. wrote the paper.

This work was supported by the Ministry of Education, Culture, Sports, Science and Technology (MEXT) KAKENHI grants 21700425 and 24700415 to M.M.; MEXT KAKENHI Grant 23500394 to J.H.; and MEXT Grants-in-Aid for Scientific Research on Innovative Areas "Memory Dynamism" (25115006) and Scientific Research (A) (25250010) to M.S. We thank Yasushi Hiromi for helpful discussions and comments, Haig Keshishian (Yale University, New Haven, (T) for GSG3285-1, Emiko Suzuki (National Institute of Genetics, Japan) for 24B-GAL4, and Takeshi Awasaki (Kyorin University, Japan) for NP6520 and NP1243. Other Drosophila lines and antibodies were obtained from the Vienna Drosophila RNAi Center, the Bloomington Stock Center, and the Developmental Studies Hybridoma Bank.

The authors declare no competing financial interests.

Correspondence should be addressed to Minoru Saitoe, Department of Sensory and Motor Systems, Tokyo Metropolitan Institute of Medical Science, 2-1-6 Kamikitazawa, Setagaya, Tokyo 156-0057, Japan. E-mail saito-mn@igakuken.or.jp.

DOI:10.1523/JNEUROSCI.3865-14.2015

Copyright $\odot 2015$ the authors $\quad 0270-6474 / 15 / 355557-09 \$ 15.00 / 0$ upon neuronal stimulation or memory formation, have not been identified.

In mammals and in other organisms, memories can be classified into various different forms. In general, short-lasting forms do not require de novo transcription and protein synthesis, while a longer lasting, consolidated form of memory, long-term memory (LTM), does (Buffington et al., 2014). Various transcription factors that are important for LTM formation have been found, and several of these, including CREB, NF- $\kappa \mathrm{B}$, and Notch, are expressed in glia as well as in neurons (Costa et al., 2005; Kaltschmidt et al., 2006; Alberini, 2009). However, previous research has focused on memory-dependent transcription/translation in neurons.

We have been studying LTM in Drosophila using a Pavlovian olfactory associative paradigm. In this system, longer lasting consolidated types of memory are formed after multiple repeated training trials. Multiple repeated trials with 15 min rest intervals between trials (spaced training) forms protein synthesisdependent LTM (Tully et al., 1994; Isabel et al., 2004), while multiple trials in succession without rest intervals (massed training) form a second type of consolidated memory, anesthesiaresistant memory (ARM). ARM can be distinguished from LTM because it does not last as long, and its formation is not suppressed by transcription or protein synthesis inhibitors (Tully et al., 1994).

Previously we demonstrated that klingon $(\mathrm{klg})$, a member of the Drosophila Ig superfamily of cell adhesion molecules (CAMs), is acutely required for LTM formation. Klg protein, but not mRNA, increases upon LTM induction (Matsuno et al., 2009). In this study, we show that Klg is required in both glia and neurons for LTM. We demonstrate that spaced training induces expres- 
sion of a glial protein, Repo, and this induction depends on Klg expression in both cell types. Repo is a glial-specific, paired-like homeodomain transcription factor, which functions to activate glial differentiation and inhibit neuronal differentiation during development (Xiong et al., 1994; Halter et al., 1995; Yuasa et al., 2003). Repo is also expressed in adult brains, but its function in adults has been unclear. We show that training-dependent increases in Repo are important for LTM formation, and that artificially elevating Repo expression can rescue the LTM defects of $\mathrm{klg}$ mutants. These results indicate that acute increases in glial gene expression are required for LTM, and further suggest that Klg-mediated communication between neurons and glia is required for this increase.

\section{Materials and Methods}

\section{Genetics, fly stocks, and constructs}

Flies were raised on standard medium at $25^{\circ} \mathrm{C}, 60 \%$ humidity, on a $12 \mathrm{~h}$ light/dark cycle, and an unselected mixture of 3- to 7-d-old male and female flies was used for all experiments in this study. $w$ (CS10), derived from outcrossing $w^{1118}$ to Canton-S for 10 generations, was used as the

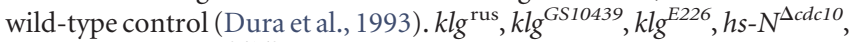
UAS-klgRNAi ( $\mathrm{klg}^{36162}$ ) and $w$; hs-GAL4/CyO (Matsuno et al., 2009); NP6520 and NP1243 (Awasaki et al., 2008), 24B-GAL4 (Brand and Perrimon, 1993), the glial-geneswitch line, GSG3285-1 (Nicholson et al., 2008); and repo ${ }^{64}$ (Yuasa et al., 2003) have all been described previously. To construct UAS-repo-myc, a full-length repo cDNA, with an added Kozak consensus sequence, and three Myc-tags fused in tandem to the $\mathrm{C}$ terminus, were cloned into the pUAST vector (Brand and Perrimon, 1993). UAS-repo ${ }^{\Delta \mathrm{AD} 302}$ and UAS-repo ${ }^{\Delta \mathrm{HD}}$-myc were generated in the same way except repo $\Delta 302$ and repo $\Delta$ box (Yuasa et al., 2003) were used in place of full-length repo. UAS-klgRNAi (III) $\left(k^{3} g^{39515}\right)$ was from the Vienna Drosophila RNAi Center, and other lines, including the UASrepoRNAi line (repo ${ }^{\text {TRiP. JF02974 }}$ ), were obtained from the Bloomington Stock Center (Indiana University). All lines, except 24B-GAL4, repo ${ }^{64}$, and repo $^{E X 84}$, were outcrossed to $w(C S 10)$ flies for at least six generations.

\section{Fly behavior}

All memory training and testing was performed in an environmental room maintained at $25^{\circ} \mathrm{C}$ and $60 \%$ humidity.

Spaced and massed training. Repetitive spaced and massed training were performed as described previously (Tully et al., 1994). Briefly, a single training session consisted of exposing $\sim 100$ flies to CS + and CS odors for $1 \mathrm{~min}$ each, where CS+ odors were paired with $1.5 \mathrm{~s}$ pulses of 60 V DC electric shocks every $5 \mathrm{~s}$, and CS- odors were not. 3-octanol and 4-methylcyclohexanol (Sigma-Aldrich) were used alternately as the CS+ and CS - odors. Spaced training consisted of 10 single-cycle trainings, with a 15 min rest interval between each training. Massed training consisted of 10 cycles of training, where one session immediately follows the previous one. After training, flies were stored in an $18^{\circ} \mathrm{C}$ incubator on a $12 \mathrm{~h}$ light/dark cycle until testing. A performance index was measured $1 \mathrm{~d}$ after training as an average of [ $(\#$ of flies choosing the CS - odor) - (\# of flies choosing the CS+ odor)]/total number of flies.

Heat-shock, RU486, and cycloheximide treatments

For heat-shock treatment, collected flies were maintained in an $18^{\circ} \mathrm{C}$ incubator at least for $3 \mathrm{~d}$ before heat shock to minimize leaky expression. Flies were then transferred to preheated vials, which were submerged in a $37^{\circ} \mathrm{C}$ water bath for $30 \mathrm{~min}$. Heat-shocked flies were returned to food vials at $25^{\circ} \mathrm{C}$ for a $6 \mathrm{~h}$ recovery period before training. For RU486 treatment, RU486 (mifepristone) was dissolved in ethanol, and mixed with fly food to a final concentration of $1 \%$ ethanol and $1 \mathrm{~mm}$ RU. Flies were fed RU486 for indicated periods of time. Cycloheximide (CXM) feeding was performed as previously described (Tully et al., 1994), except that flies were transferred to vials containing strips of Whatman filter paper soaked with $35 \mathrm{~mm} \mathrm{CXM}$ and $5 \%$ sucrose for $16 \mathrm{~h}$ before training.

\section{Histology}

For immunohistochemistry, $10 \mu \mathrm{m}$ frontal paraffin sections of heads were cut and processed as previously described (Skoulakis and Davis,
1996; Crittenden et al., 1998). Antigens in paraffin sections were reactivated by heating for $10 \mathrm{~min}$ at $95^{\circ} \mathrm{C}$ in $10 \mathrm{~mm}$ sodium citrate, $\mathrm{pH} 6.0$. Immunocytochemistry of cultured cells was performed as previously described (Su and O'Dowd, 2003).

Two rabbit anti-Klg polyclonal antibodies were generated, anti-Klg-1, against a 20-mer peptide, PVPSIYWTKKSGANKSTARI, and anti-Klg-2, against a 26-mer peptide, CKGSGNPVPSIYWTKKSGANKSTARI, from the second Ig domain of the Klg protein. Antibodies were affinity purified using their peptide antigens, and both purified antibodies recognized the same size Klg band as described previously (Butler et al., 1997). AntiKlg-2 antibody was used for immunohistochemistry and Western blots, and anti-Klg-1 antibody was used for immunocytochemistry experiments. Purified anti-Klg-1 was used at 1:50, purified anti-Klg-2 was used at 1:10, chick anti-GFP (Abcam) was used at 1:500, and mouse anti-Repo 8D12 (Developmental Studies Hybridoma Bank) was used at 1:20 for histology experiments. Topro-3 (Invitrogen) was used at $0.5 \mu \mathrm{M}$. The numbers of Repo-positive glia and the intensities of Repo staining were calculated using ImageJ (NIH; http://rsbweb.nih.gov/ij/).

\section{Cell culture}

Primary pupal CNS cells were cultured as described previously (Miyashita et al., 2012). Cells were placed on concanavalin A laminin-coated glass coverslips and cultured in a $23^{\circ} \mathrm{C}$ humidified $5 \% \mathrm{CO}_{2}$ incubator. Four-day-old cultured cells were used for antibody staining.

\section{Western blotting}

Affinity-purified anti-Klg-2 antibody (1:20), monoclonal antibody against Repo (1:20; 8D12 from Developmental Studies Hybridoma Bank), and monoclonal anti-myc antibody (1:1000; 9B11 from Cell Signaling Technology) were used for Western quantification of Klg and Repo. Mouse anti- $\alpha$-tubulin antibody (1:1000, \#DM1a from Seikagaku Kogyo) was used for normalization. Head extracts were made in homogenization buffer [ $25 \mathrm{~mm}$ HEPES, $100 \mathrm{~mm} \mathrm{NaCl}, 1 \mathrm{~mm} \mathrm{MgCl}_{2}, 1 \mathrm{~mm}$ $\mathrm{CaCl}_{2}, 0.2 \%$ Trion X-100, 0.2\% Nonidet P-40, and protease inhibitors (Roche)]. Signals were detected using HRP-conjugated secondary antibodies and ECL blotting reagents (GE Healthcare). Protein amounts were quantified using ImageJ.

\section{Quantification of transcripts by quantitative PCR}

Total RNA from Drosophila heads was extracted using TRIzol reagent (Invitrogen), and cDNA was synthesized using RevTraAce (Toyobo) as described previously (Matsuno et al., 2009). Transcript amounts were quantified using real-time PCR (model 7500; Applied Biosystems). Expression of rp49 was used for normalization since rp49 amounts remain unchanged after spaced training (Miyashita et al., 2012). Primers used were as follows: repo-F: 5'-ACCATTTGGATCATGGCAGT-3'; repo-R: 5'-AACACATCTCATCGGGCTTC-3'; rp49-F: 5'-AGCATACAGGCCCAAGATCGTG-3'; and rp49-R: 5' ${ }^{\prime}$-CGACAGCTTAGCATATCGAT CC- $3^{\prime}$.

\section{Statistics}

All data in bar graphs are means \pm SEMs. Sample sizes are indicated within each bar in all graphs. Data were analyzed using the Student's $t$ test for comparisons between two groups, and one-way and two-way ANOVA followed by indicated post hoc tests for multiple analyses. $P \leq$ 0.05 was considered statistically significant. Analyses were performed using Excel 2011 (Microsoft) with add-in software and Prism version 6.0 (GraphPad).

\section{Results}

\section{Both neuronal and glial expression of Klingon $(\mathrm{klg})$ is required for LTM formation}

We previously found that klingon $(k l g)$ is acutely required for olfactory LTM, and amounts of Klg protein increase upon LTM induction (Matsuno et al., 2009). To determine where Klg is localized in the Drosophila brain, we generated antibodies and observed Klg using immunohistochemistry. We observed discreet, punctate Klg signals throughout the brain in a pattern surrounding neuropil structures (Fig. $1 A$ ). This pattern suggested that $\mathrm{Klg}$ 

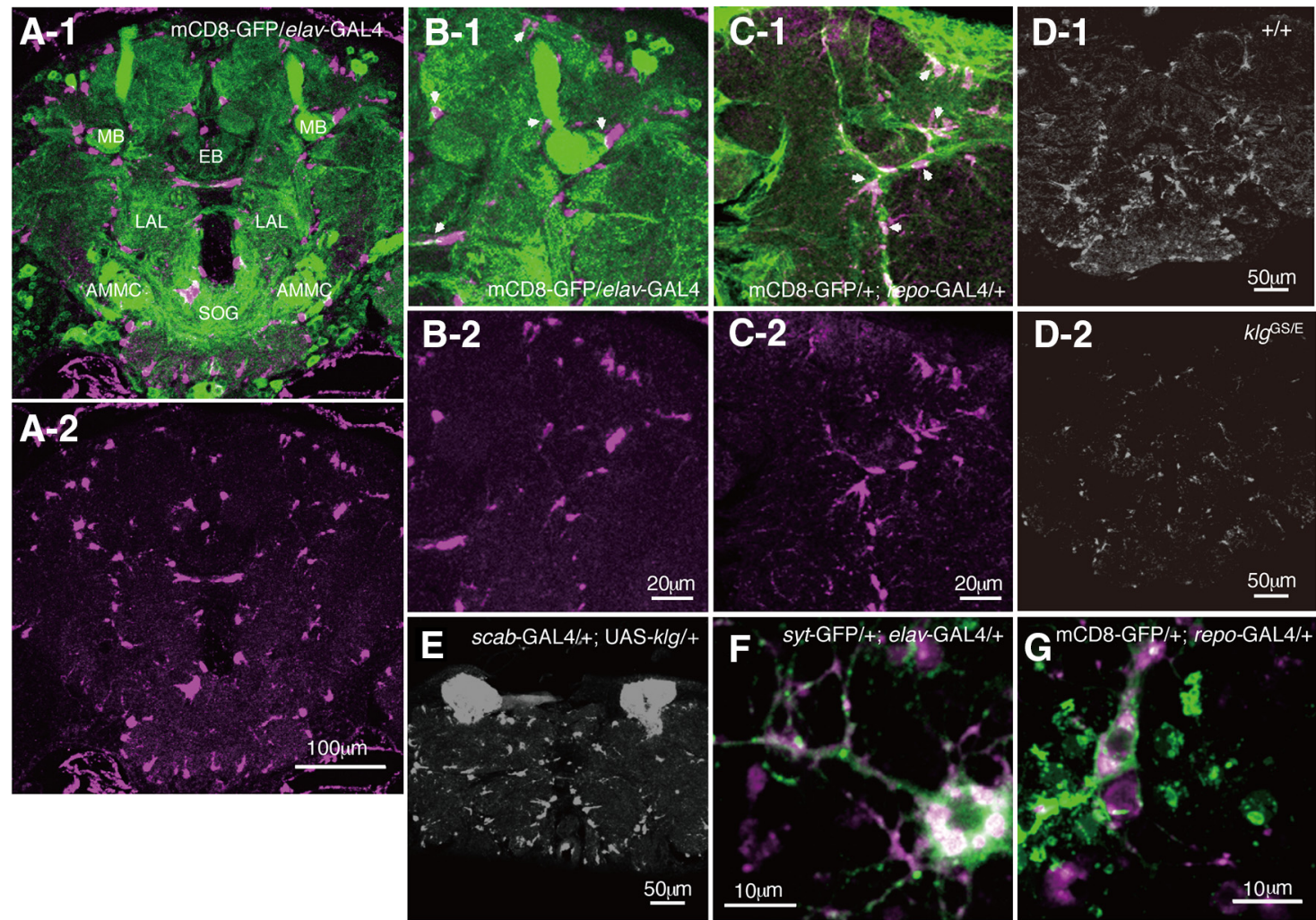

H
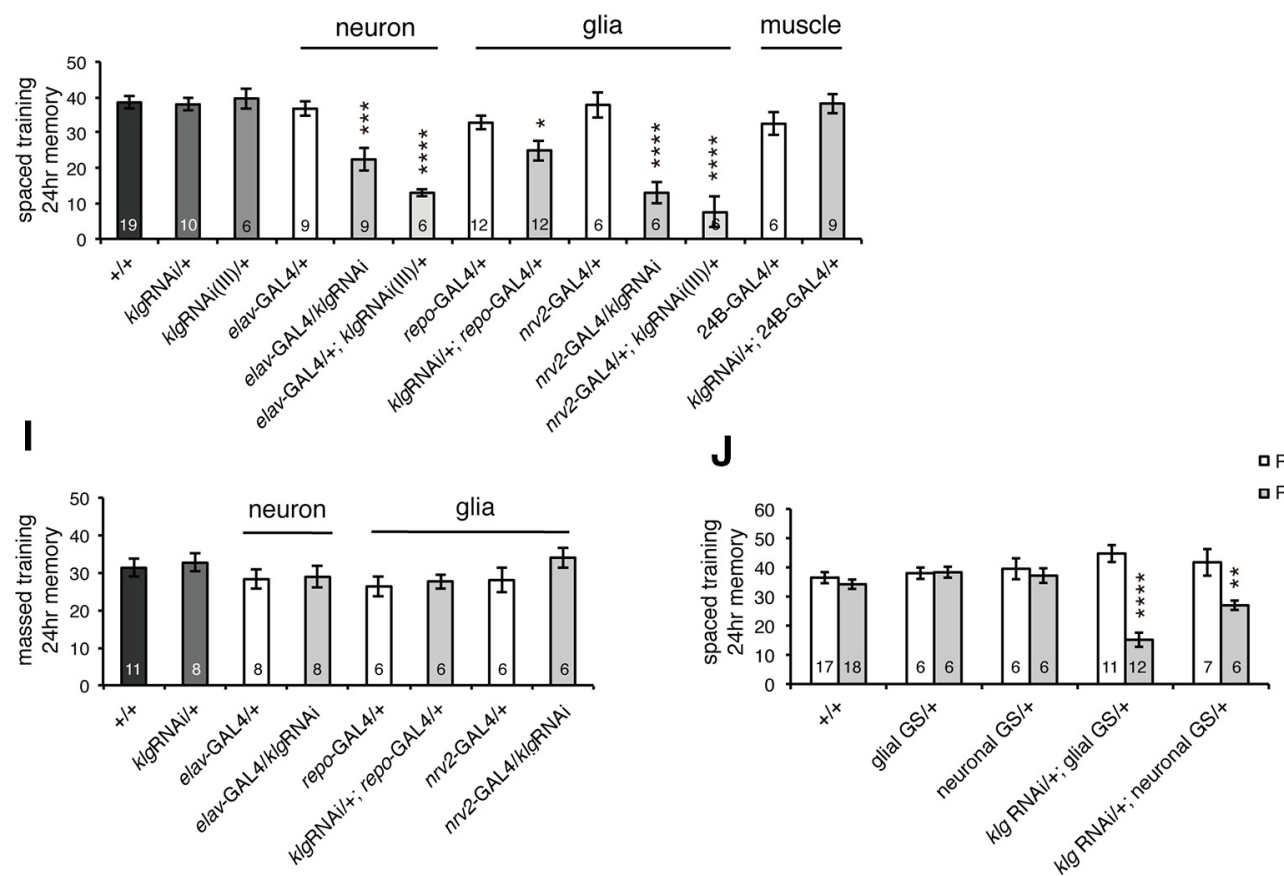

Figure 1. Klingon ( $\mathrm{klg}$ ) expression in both neurons and glia is required for LTM. A, Klg immunostaining of an adult-head paraffin slice. A-1, Klg (magenta) is observed in a punctate pattern surrounding neuropil regions. GFP expressed in neurons (elav-GAL4/UAS-mCD8-GFP) is shown in green. EB, ellipsoid body; LAL, lateral accessory lobes; AMMC, antennal mechanosensory and motor center; SOG, subesophageal ganglion. $\boldsymbol{A}-\mathbf{2}$, Klg immunostaining alone. $\boldsymbol{B}-\mathbf{1}, \boldsymbol{B}-\mathbf{2}$, Higher magnification images of $\boldsymbol{A}-\mathbf{1}$ and $\boldsymbol{A}-\mathbf{2}$ that show that Klg and neuronal GFP staining patterns partially overlap (indicated by white arrows). C, Klg and GFP staining partially overlap when GFP is expressed in glia (UAS-mCD8-GFP/+; repo-GAL4/+). C-1, Staining with anti-Klg (magenta) and anti-GFP (green) antibodies. Areas of overlap are indicated with white arrows. C-2, Klg immunostaining alone. D, Klg amounts in wild-type (D-1) and in $\mathrm{klg}{ }^{\mathrm{GS} 10439} / \mathrm{klg} g^{\mathrm{E226}}(\mathbf{D}-2)$ flies. Compared with wild-type flies, $\mathrm{klg}^{G 510439} / \mathrm{klg}^{E 226}$ flies show reduced Klg immunoreactivity. E, Klg amounts in scab-GAL4/+; $k 1 g^{G 510439} /+$ flies. When $\mathrm{klg}$ is expressed in the MBs under $\alpha$-integrin (scab) promoter control, increased Klg immunoreactivity is observed in the MB lobes (data not shown) and calyces. $\boldsymbol{F}, \mathbf{G}$, Klg protein localization in Drosophila primary cell cultures. $\boldsymbol{F}$, Partial overlap of Klg and synaptotagmin-GFP (syt-eGFP) signals in primary pupal CNS cultures from UAS-syt-GFP/+; elav-GAL4/+ brains. A subset of neurons expresses Klg (magenta). G, Partial overlap of Klg and GFP signals in primary pupal CNS cultures from UAS-mCD8-GFP/+; repo-GAL4/+ brains. Klg (magenta) is found in a subset of glia. $\boldsymbol{H}$, klg knockdown in neurons and glia, but not muscles, inhibits LTM. One-way ANOVA indicates significant differences in memory between genotypes $\left(F_{(12,103)}=15.91, p<0.0001\right) .{ }^{*} p<0.05 ;{ }^{* * *} p<0.001 ;{ }^{* * * *} p<0.0001$ versus both GAL4-alone and (Figure legend continues.) 
may be expressed on or near glia, and we examined colocalization between Klg and GFP expressed in either neurons (from an elavGal4 driver) or in glia (from a repo-Gal4 driver). Interestingly, we observed both overlapping and nonoverlapping Klg and GFP signals when GFP was expressed in either cell type (Fig. 1B,C), indicating that $\mathrm{Klg}$ is expressed in both neurons and glia, and may localize to the juncture between the two. We observed reduced Klg signals in brains of $\mathrm{klg}^{G S 10439} / \mathrm{klg}^{E 226}$ flies (Fig. 1D), and increased signals in the mushroom bodies (MBs) when $\mathrm{klg}$ was ectopically expressed using an MB driver (Fig. 1E, scab-GAL4/+; $\mathrm{klg}^{G S 10439} /+$ flies), confirming the specificity of our antibody. To observe the cellular localization of Klg protein in more detail, we stained Drosophila primary cell cultures with Klg antibody and found that Klg is expressed in subsets of both neurons and glia (Fig. $1 F, G$ ). Since Klg is a CAM that forms homophilic interactions, this suggests that Klg may mediate neuron glial interactions required for LTM.

To determine whether $k l g$ needs to be expressed in neurons, glia, or in both cell types for LTM, we next expressed $k l g$-RNAi constructs from cell type-specific drivers. Knockdown of $\mathrm{klg}$ in both neurons and glia, using two independent $k l g$-RNAi lines, inhibited LTM, whereas knockdown of $k l g$ in muscle had no effect (Fig. 1H). We previously showed that Klg is not required for a second type of consolidated memory, ARM, which is formed after massed training. Consistent with this, klg knockdown in neurons and glia had no effect on memory after massed training (Fig. 1I).

To establish whether Klg is required during development, or acutely at the time of memory formation, we expressed $k l g$-RNAi from RU486-inducible glial-GeneSwitch-Gal4 (GSG3285-1; Nicholson et al., 2008) or neuronal-GeneSwitch-Gal4 (elav-GS) drivers. Feeding adult flies $1 \mathrm{~mm}$ RU486, from $3 \mathrm{~d}$ before training until testing, disrupted LTM in both $\mathrm{klg}-\mathrm{RNAi} /+$; glial-GS/+ and $\mathrm{klg}$ $R N A i /+$; elav-GS/+ flies, compared with nonfed controls (Fig. $1 J)$.Our results suggest that an acute increase in Klg-mediated adhesion between neurons and glia after training is required for formation of LTM but not ARM.

Klg regulates amounts of the glial transcription factor, Repo To further understand the function of Klg, we next characterized glial changes in $k l g$ mutants compared with wild-type. We first quantified glial cell numbers in wild-type and mutants by visualizing glia in head paraffin sections using antibody to the glial nuclear marker, Repo (Fig. $2 A, B$ ). Although we did not find a difference in numbers of glial cells, we noticed a significant decrease in intensity of Repo nuclear staining in $\mathrm{klg}$ mutants (Fig. 2C). We confirmed that Repo amounts are decreased in $\mathrm{klg}$ mutant and $\mathrm{klg}$-knockdown heads by Western blotting (Fig. 2D). Previously we reported that $\mathrm{klg}$ expression is regulated by the Notch signaling pathway, and that Klg amounts are reduced upon expression of a dominant-negative

(Figure legend continued.) UAS-alone controls as assayed by Fisher's least significant difference tests. repo-GAL4 and nrv2-GAL4 express GAL4 in glia, elav-GAL4 expresses GAL 4 in neurons, and 24B-GAL4 expresses GAL4 in muscle. I, Knockdown of $\mathrm{klg}$ in neurons and glia does not affect ARM. One-way ANOVA did not identify significant differences between genotypes $\left(F_{(7,51)}=\right.$ $0.9758, p=0.4589)$. J, Acute knockdown of $\mathrm{klg}$ in both neurons and glia inhibits LTM. klg was knocked down by expressing klg RNAi from neuronal and glial geneswitch drivers, and feeding flies $1 \mathrm{~mm}$ RU486 for $4 \mathrm{~d}$ at the adult stage. Two-way ANOVA identified significant differences due to genotype $\left(F_{(4,85)}=3.435, p=0.0119\right)$, RU feeding $\left(F_{(1,85)}=30.56, p<0.0001\right)$, and interaction between genotype and RU feeding $\left(F_{(4,85)}=13.49, p<0.0001\right)$. ${ }^{* *} p<0.01$ and ${ }^{* * * *} p<0.0001$ compared with RU - controls, assayed by Bonferroni post hoc tests.
Notch construct, $N^{\Delta c d c 10}$ (Matsuno et al., 2009). Consistent with this, we found that acute induction of $N^{\Delta c d c 10}$ also reduces Repo amounts (Fig. 2E). To determine whether Klg is regulated by Repo, we measured Klg protein in repo mutants and repo knockdown lines (Fig. $2 F$ ), and in transgenic flies overexpressing myc-tagged repo ${ }^{+}$or a transcription-deficient version (repo $\Delta H D-m y c$; Fig. $2 G$ ). We observed no significant changes in Klg amounts upon Repo manipulations, indicating that Klg regulates Repo, but not the converse. Interestingly, knockdown of $\mathrm{klg}$ in both neurons and glia caused similar decreases in Repo (Fig. 2D), indicating that neuronal Klg exerts a non-cell autonomous effect on glial Repo.

\section{Repo is required for LTM formation}

Since Klg regulates Repo, we next examined whether Repo is required for LTM formation, and found that heterozygous repo loss-of-function mutants have significantly decreased $1 \mathrm{~d}$ memory after spaced training (Fig. 3A, left). This decrease consists of defects in LTM since feeding of a protein synthesis inhibitor, CXM, before training did not further reduce memory in mutants (Fig. $3 A$, right). One day memory after massed training is normal in both CXM-fed and control-fed repo mutants (Fig. 3B), indicating that repo mutants are defective for LTM, but not ARM, a phenotype similar to $\mathrm{klg}$ mutants. To address whether repo expression at the adult stage is required for LTM formation, we next acutely inhibited expression using a heat shock-inducible repo RNAi line. Repo protein amounts were significantly reduced $6 \mathrm{~h}$ after a $30 \mathrm{~min} 37^{\circ} \mathrm{C}$ heat shock in $h s$-GAL4/UAS-repoRNAi flies (Fig. $2 F$ ). The same heat-shock regimen performed $6 \mathrm{~h}$ before spaced training also significantly reduced $1 \mathrm{~d}$ memory in these flies, while it had no effect on control flies (Fig. $3 C$ ). To discount the possibility of off-target effects of repoRNAi, we also inhibited endogenous Repo function by expressing a dominant-negative repo construct (repo ${ }^{\triangle A D 302}$, containing a large deletion in its transcriptional activation domain), under control of the RU486inducible glial-GS promoter. We found that feeding of $1 \mathrm{~mm}$ RU486, from $3 \mathrm{~d}$ before spaced training until testing, specifically disrupted $1 \mathrm{~d}$ memory in glial-GS $>$ repo ${ }^{D N}$ flies while it had no effect on controls (Fig. 3D).

In Drosophila, several different classes of glia have been characterized, including two types of neuropil glia: ensheathing glia, which are proposed to provide insulation between neighboring neural compartments, and astrocyte-like glia, which are thought to potentially associate with synapses (Awasaki et al., 2008; Doherty et al., 2009; Awasaki and Lee, 2011). To determine whether repo expression in a specific type of neuropil glia is required for LTM, we next knocked down repo in either ensheathing glia (using the NP6520 driver) or in astrocyte-like glia (from the NP1243 driver), and measured effects on $24 \mathrm{~h}$ memory after spaced training. We observed significant reductions when repo was knocked down in astrocyte-like glia compared with controls, while there was no difference when repo was knocked down in ensheathing glia compared with driver alone (Fig. $3 E$ ). Our results suggest that Repo-dependent gene expression is required acutely in astrocytelike glia during LTM formation.

\section{Training-dependent increase in Repo is required for Klg- dependent LTM formation}

During glial development, Repo is activated in differentiating glial cells through an increase in expression (Lee and Jones, 2005). To determine whether a similar type of regulation also occurs during memory formation, we next measured Repo amounts after training, and found a significant increase in Repo protein $24 \mathrm{~h}$ 

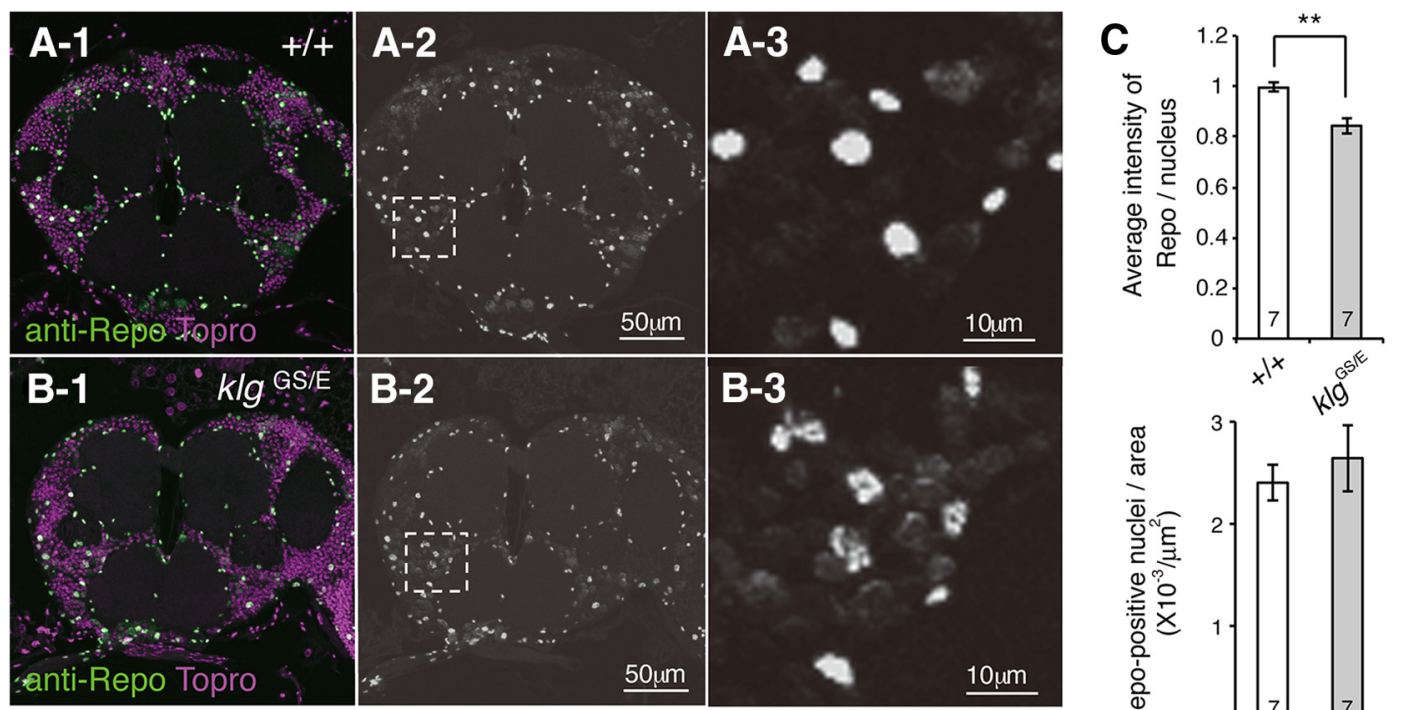

D
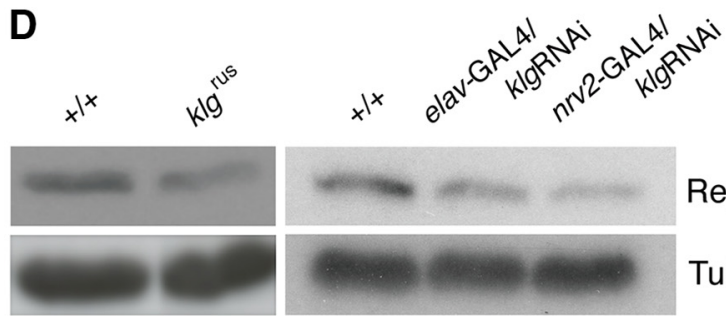

Repo

Tub

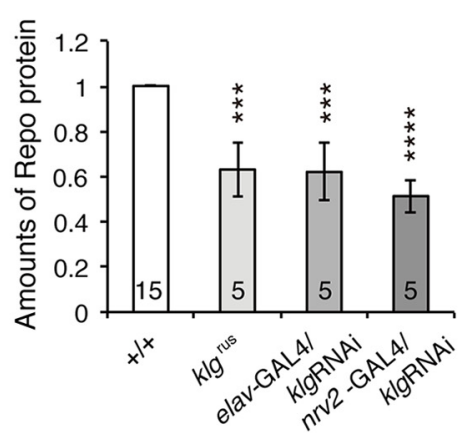

F

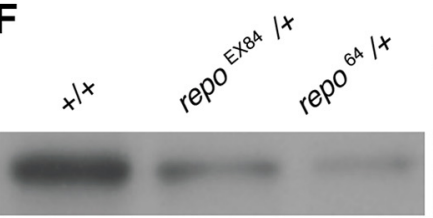

hs-GAL4/+; repoRNAi/+

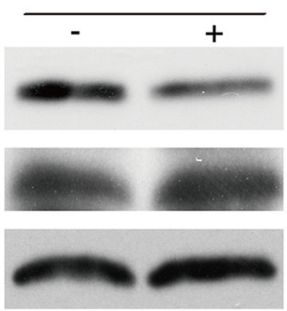

E

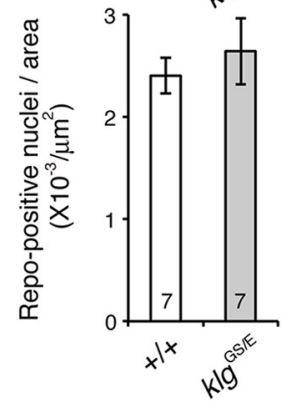

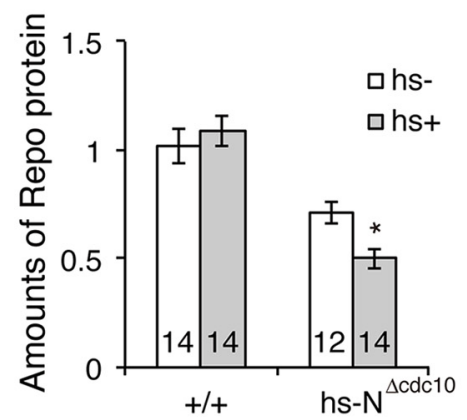

hs

Repo

Klg

Tub

G repo $\Delta$ HD-myc/+; repo -myc/t;

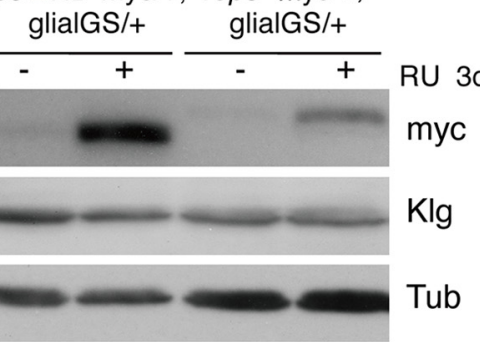

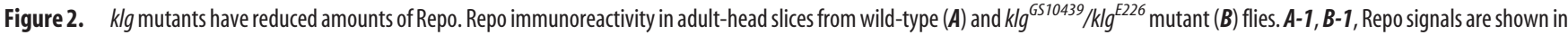
green, and nuclei (labeled with Topro) are shown in magenta. $\boldsymbol{A}-\mathbf{2}, \boldsymbol{B}-\mathbf{2}$, Black and white images of Repo immunoreactivity. $\boldsymbol{A}-\mathbf{3}, \boldsymbol{B}-\mathbf{3}$, Higher magnification images of the boxed regions in $\boldsymbol{A}-\mathbf{2}$ and $\boldsymbol{B}$-2. C, Top, Quantification of the average Repo signal intensity/nucleus from seven wild-type $(+/+)$ and $k l g 610439 / \mathrm{klg}^{E 226}$ mutant heads. Wild-type intensity was normalized to 1 . ${ }^{* *} p<0.01$ as assayed by $t$ test. Bottom, The number of Repo-positive nuclei/area is unaffected in $\mathrm{klg} \mathrm{g}^{610439} / \mathrm{klg} \mathrm{g}^{\mathrm{E} 226}$ mutants. D, Top, Representative western blot showing amounts of Repo in head extracts from wild-type $(+/+), \mathrm{klg}^{\text {rus }}$, and neuronal and glial klg knockdown lines (elav-GAL4/UAS-klg RNAi and nrv2-GAL4/UAS-klg RNAi). $\alpha$-Tubulin (Tub) amounts are shown for comparison. Bottom, Quantification of western blot data. One-way ANOVA indicates significant differences between genotypes $\left(F_{(3,26)}=16.02, p<0.0001\right)$. ${ }^{* *} p<0.001$ and ${ }^{* * * *} p<0.0001$ versus $+/+$ as assayed by Dunnett's multiple-comparison tests. $\boldsymbol{E}$, Repo amounts decrease upon heat shock-dependent expression of dominant-negative Notch. Acute heat shock, $6 \mathrm{~h}$ before harvesting, reduces Repo in $h s-N^{\Delta c d c 10}$, but not wild-type heads. Two-way ANOVA identifies significant differences due to genotype $\left(F_{(1,50)}=50.46, p<0.0001\right)$, and interaction between heat shock and genotype $\left(F_{(1,50)}=\right.$ $4.991, p=0.03)$. Bonferroni post hoc comparisons demonstrate a significant difference in Repo amounts after heat shock in $h s-N^{\Delta c d c 10}$ heads $\left({ }^{*} p<0.05\right)$, but no significant difference in wild-type. F, Western blots showing Repo, Klg, and Tubulin (Tub) amounts in wild-type $(+/+)$, repo ${ }^{\mathrm{Ex} 84} /+$, repo ${ }^{64} /+$, and hs-GAL4/+; repoRNAi/+ head extracts. G, Western blots showing amounts of myc-tagged Repo, Klg, and Tubulin upon acute RU486-induced overexpression of myc-tagged repo or myc-tagged mutant repo (repo $\Delta H D$ ) in glial cells. 

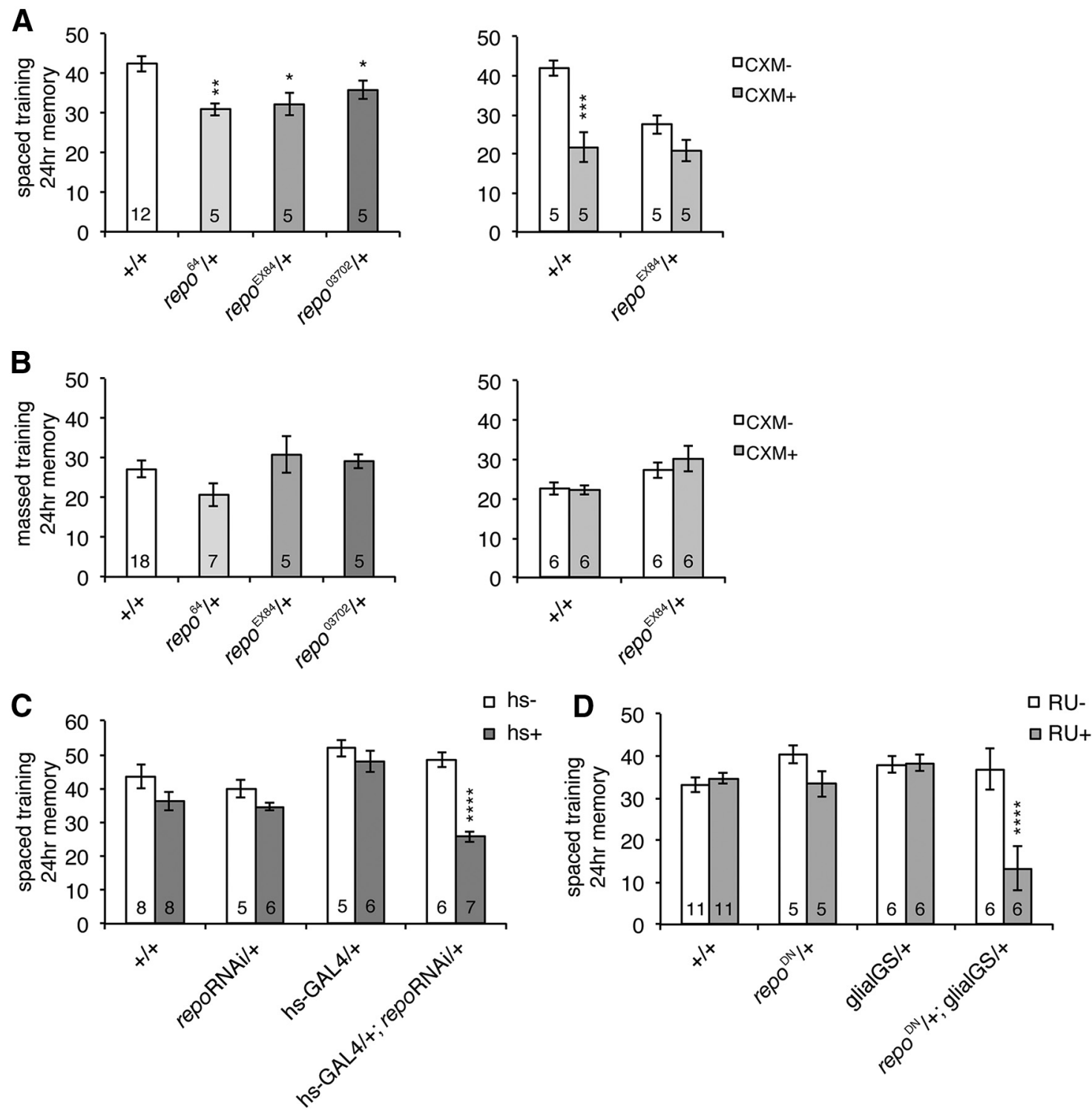

E

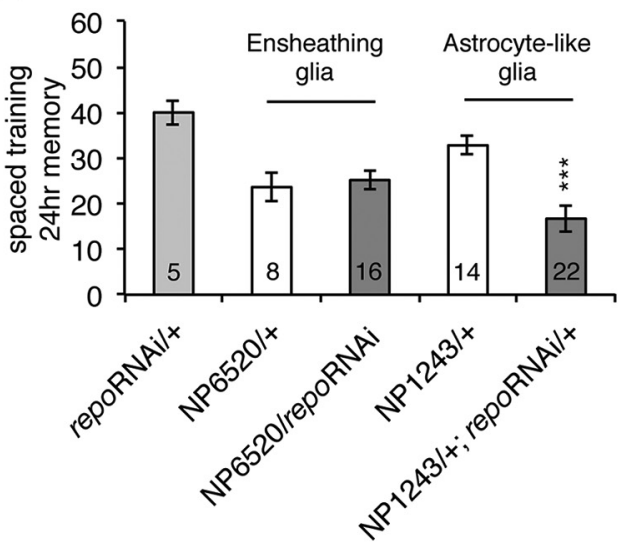

Figure 3. Repo is required for LTM formation. $A$, Left, repo hypomorphic mutants are defective for memory $24 \mathrm{~h}$ after spaced training. Experiments were performed comparing single mutants to wild-type. ${ }^{*} p<0.05 ;{ }^{* *} p<0.01$ compared with $+1+$ by $t$ test. Right, $\mathrm{XXM}$ treatment does not reduce $24 \mathrm{~h}$ memory in repo mutants. Two-way ANOVA indicates significant differences due to genotype $\left(F_{(1,16)}=7.448, p=0.0149\right)$, CXM feeding $\left(F_{(1,16)}=22.90, p=0.0002\right)$, and interaction between genotype and $C X M$ feeding $\left(F_{(1,16)}=5.639, p=0.0304\right)$. Bonferroni post hoc tests reveal significant differences due to CXM feeding in wild-type ( ${ }^{* * *} p<0.001$ ), but not repo ${ }^{E X 84}$ mutants. $B$, Left, $24 \mathrm{~h}$ memory formed after massed training. Right, Effects of $C X M$ feeding on $24 \mathrm{~h}$ memory after massed training. C, Acute knockdown of repo disrupts LTM. A $37^{\circ} \mathrm{C}$ heat shock (hs + ) for 30 min significantly disrupted $1 \mathrm{~d}$ memory after spaced training in $h s-G A L 4 /+$; UASrepoRNAi/+ flies. Two-way ANOVA demonstrates significant differences due to genotype $\left(F_{(3,43)}=9.189, p<0.0001\right)$, heat-shock regimen $\left(F_{(1,43)}=26.90, p<0.0001\right)$, and interaction between genotype and heat shock $\left(F_{(3,43)}=5.245, p=0.0036\right) .{ }^{* * *} p<0.0001$ compared with hs-, determined by Bonferroni post hoc tests. $D$, Acute overexpression of (Figure legend continues.) 
after spaced, but not massed, training (Fig. 4A). We also observed an increase in repo mRNA $24 \mathrm{~h}$ after spaced training (Fig. 4B), indicating that increases in Repo are likely to occur at the level of transcription.

Spaced training-dependent increases in Repo parallel the spaced training-dependent increases in Klg protein that we reported previously (Matsuno et al., 2009). Thus, we next tested whether the increase in repo expression depends on Klg. Consistent with the results shown in Figure 2, Repo amounts in naive flies are reduced $\sim 2$-fold in $k l g$ mutants and knockdown flies (Fig. 4C). In addition, spaced training-dependent increases are completely abolished in these mutants and knockdown flies, suggesting that training-dependent increases in Klg-mediated neuron glia adhesion are responsible for increasing Repo expression.

Since Repo is required for normal LTM, we next considered the possibility that reduced Repo could be the cause of LTM defects in $k l g$ mutants. Thus we measured LTM in $k l g$ knockdown flies and in knockdown flies expressing a repo transgene in glia. As seen in Figure $4 D$, glial expression of a wild-type repo transgene is sufficient to rescue the LTM defects of $k l g$ knockdown flies. Expression of a nonfunctional repo transgene lacking its homeobox DNA-binding domain (repo $\Delta H D$ ), or expression of an unrelated protein, GFP, does not rescue these defects, indicating that rescue depends specifically on transcriptionally active Repo. These results suggest that training-dependent strengthening of neuron glia adhesion increases expression of glial Repo, and this increase is sufficient for normal LTM.

\section{Discussion}

Many changes in gene expression during LTM induction and long-term plasticity have been characterized, including activation of various immediate early genes (Alberini, 2009). Although several of these genes are expressed in both glia and neurons, data showing activity-dependent changes in expression specifically in glia have not been previously reported, and most plasticity models focus on changes in neurons. Here we show that spaced training increases repo gene expression and protein amounts, and that LTM requires Repo activity. Repo functions during development to activate glial differentiation and inhibit neuronal differentiation, but it is also expressed in adults, and is used extensively as a glial marker. Our data indicate that an acute increase in glial transcription occurs to increase Repo amounts during LTM formation. Since Repo itself is a transcriptional activator, our data further suggest that a cascade of glial transcription may be required during LTM formation.

In neurons, various steps are involved in inducing LTMassociated transcription ( Pláteník et al., 2000). Neuronal activity induces postsynaptic depolarization, resulting in $\mathrm{Ca}^{2+}$ influx,

\section{$\leftarrow$}

(Figure legend continued.) dominant-negative repo in glia disrupts LTM. The glial-GS driver was used to overexpress dominant-negative repo in the presence of RU486 in a UAS-repo ${ }^{\triangle A D 302} /+$; glial-GS-GAL4/+ line. RU486 (1 mM) was fed to flies from $3 \mathrm{~d}$ before training until testing. Two-way ANOVA indicates significant differences due to genotype $\left(F_{(3,48)}=7.347, p=\right.$ $0.0004)$, RU feeding $\left(F_{(1,48)}=11.81, p=0.0012\right)$, and interaction between genotype and feeding $\left(F_{(3,48)}=8.020, p=0.0002\right) .{ }^{* * * *} p<0.0001$ compared with $\mathrm{RU}-$, as determined by Bonferroni post hoc tests. $\boldsymbol{E}$, repo knockdown in astrocyte-like glia (NP1243/+; repo RNAi/+) disrupts LTM, while knockdown in ensheathing glia (NP6520/repoRNAi) does not. One-way ANOVA indicates significant differences due to genotype $\left(F_{(4,60)}=7.979, p<\right.$ 0.0001). Tukey's multiple-comparison tests reveal significant differences between NP1243/+; repo RNAi/+ and both repoRNAi/+ and NP1243/+ controls ( $\left.{ }^{* * *} p<0.001\right)$, but no significant differences between NP6520/repoRNAi and the NP6520/+ control. and activation of second messengers. Second messengers, in turn activate pre-existing transcription factors, which then induce expression of downstream effector genes, including additional transcription factors. While the steps involved in inducing glial gene expression are still unclear, we demonstrate that LTMassociated increases in Repo require the CAM, Klg. Klg amounts, on the other hand, are unaffected by alterations in Repo activity, indicating that Repo functions downstream of Klg. Supporting this idea, glial expression of a repo transgene is sufficient to rescue $\mathrm{klg}$ LTM defects, suggesting that the primary function of Klg during LTM formation is to induce training-dependent glial repo expression. Klg is found at the juncture between neurons and glia, and needs to be expressed in both of these cell types to induce repo expression and LTM formation. Thus neuronal Klg functions non-cell autonomously, while glial Klg functions cell autonomously, to induce repo. Since Klg is a cell adhesion that forms homophilic interactions, we propose that neuron-glia Klg interactions transmit information about neuronal activity to glia. We have previously shown that amounts of Klg protein, but not mRNA, increase upon LTM induction (Matsuno et al., 2009). This increase may directly enhance neuron glial signaling, or alternatively, may enhance neuron glial adhesion, causing increased repo expression through an indirect mechanism.

We do not believe that Repo functions at the apex of a transcription cascade during LTM formation because expression of repo itself increases after spaced training. Instead, we propose that a pre-existing glial transcription factor is activated by Klg, and increases repo expression. Repo then functions as a downstream effector molecule, regulating expression of a variety of glial genes further downstream that are required for LTM.

Previous studies have tried to identify where proteins are synthesized during LTM formation. In particular, Chen et al. (2012) did not observe a decrease in LTM when protein synthesis was acutely inhibited in repo-expressing cells. While these data seem inconsistent with ours, when Chen et al. (2012) inhibited protein synthesis using a second glial driver derived from the crammer gene, LTM was severely impaired. Thus it is possible that the repo driver used by Chen et al. (2012) may not have inhibited synthesis enough to affect LTM.

What downstream genes does Repo control? Learninginduced changes in nuclear RNA were reported $>50$ years ago (Hyden and Egyhazi, 1962), and these occur in both neurons and glia. Surprisingly, however, very few glial effector genes showing altered expression upon memory formation have been identified. In mammals, amounts of a glial $\mathrm{Ca}^{2+}$ binding protein, $\mathrm{S} 100 \beta$, have been reported to increase upon memory formation (Hydén and Lange, 1970), while in Drosophila, expression of crammer (cer), which encodes an inhibitor of the cathepsin family of cysteine proteinases, has been reported to decrease after spaced training (Comas et al., 2004). cer is found in both neuropil and glia, but it is thought to exert an effect on LTM in glia. A Drosophila homolog to $S 100 \beta$ has not been identified, and decreased cer expression occurs $3 \mathrm{~h}$ after training, before Repo increases. This suggests that $S 100 \beta$ and cer are not regulated by Repo. Recently, various genes regulated by Repo have been identified, and several of these, including members of the wnt/wingless signaling pathway, have been shown to affect synaptic structure and efficacy (Kerr et al., 2014). It will be of great interest to determine whether these or other genes are also components of a training-induced glial transcription pathway required for LTM. 
A

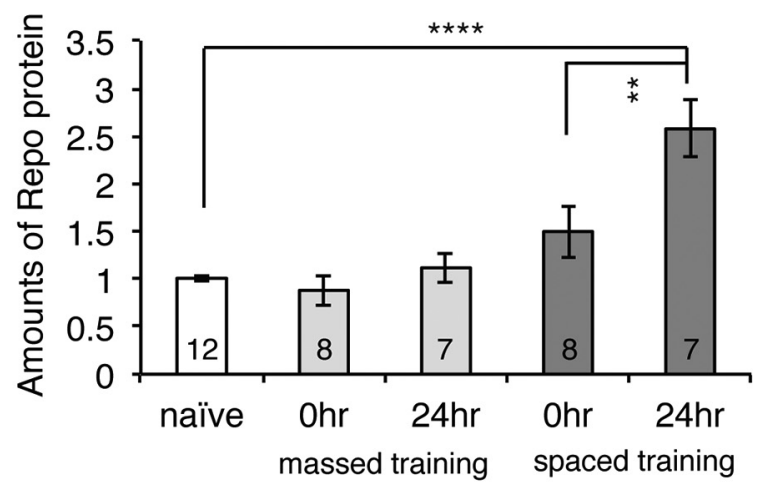

B

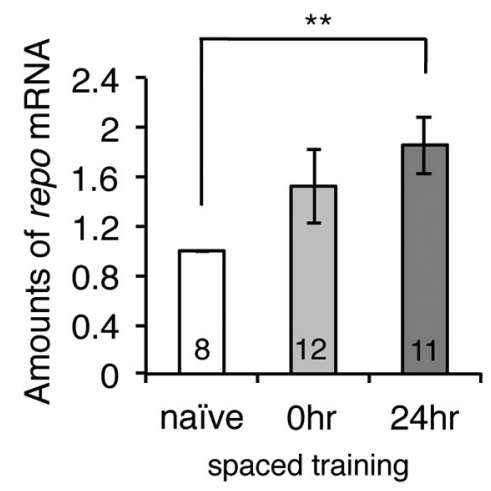

C

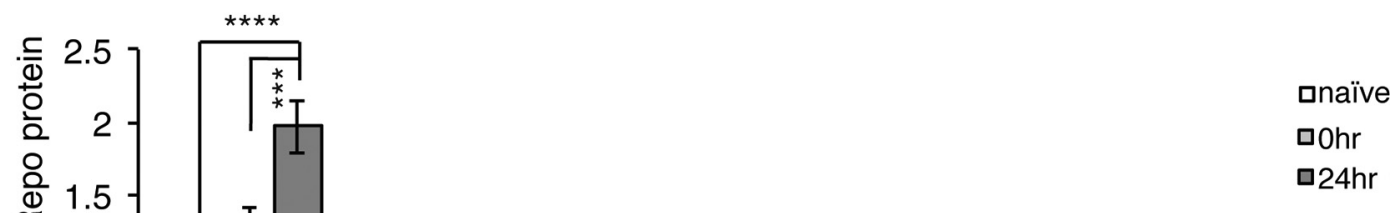

D

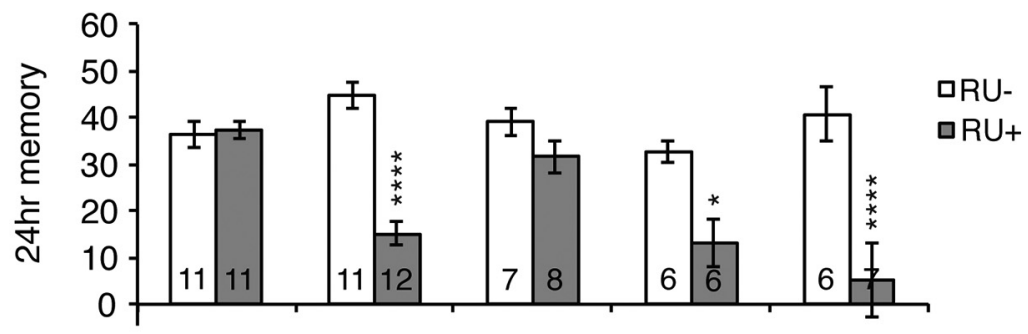

$\begin{array}{rrrrrr}\text { glial GS } & - & + & + & + & + \\ \text { UAS-kIgRNAi } & - & + & + & + & + \\ \text { UAS-repo+ } & - & - & + & - & - \\ \text { UAS-repoLHD } & - & - & - & + & - \\ \text { UAS-GFP } & - & - & - & - & +\end{array}$

Figure 4. Repo amounts increase during LTM formation, and increasing repo expression is sufficient to rescue $k \mathrm{lg}$ LTM defects. $A$, Amounts of Repo (normalized to $\alpha$-tubulin) in fly heads increase after spaced training (one-way ANOVA, $F_{(2,24)}=16.63, p<0.0001$ ), but not after massed training (one-way ANOVA, $F_{(2,24)}=1.025, p=0.3740$ ). The increase in Repo becomes significant $24 \mathrm{~h}$ after training. ${ }^{* * *} p<0.0001$ and ${ }^{* *} p<0.01$ as assayed by Tukey's post hoc tests. B, Amounts of repo mRNA also increase after spaced training. ${ }^{* *} p<0.01$ versus naive by $t$ test. C, Spaced training-dependent increases in Repo protein are not seen in $\mathrm{klg}^{\mathrm{rus}}, \mathrm{klg}^{G 510439} / \mathrm{klg}^{\text {E226, }}$, and neuronal (elav-GAL4/UAS-klg RNAi) and glial (nrv2-GAL4/UAS-klg RNAi) klg knockdown lines. Two-way ANOVA indicates significant differences due to genotype $\left(F_{(4,102)}=14.51, p<0.0001\right)$, time after training $\left(F_{(2,102)}=3.197, p=0.045\right)$, and interaction between genotype and time after training $\left(F_{(8,102)}=2.180\right.$, $p=0.035) .{ }^{* * *} p<0.0001$ and ${ }^{* * *} p<0.001$ as determined by Bonferroni post hoc tests. $\boldsymbol{D}$, Glial expression of repo is sufficient to rescue LTM defects of klg knockdown lines. Knockdown of klg using a glial-GeneSwitch driver (UAS-klg RNAi/+; glial-GeneSwitch-GAL4/+) in the presence of RU486 disrupts LTM. Glial expression of repo (UAS-klg RNAi/UAS-repo ${ }^{+}$; glial-GeneSwitch-GAL4/+) is sufficient to rescue this defect, while expression of an unrelated protein, GFP (UAS-klg RNAi/UAS-GFP; glial-GeneSwitch-GAL4/+), or expression of transcriptionally inactive repo lacking its homeodomain (UAS-klg RNAi/UASrepo $\Delta H D$; glial-GeneSwitch-GAL4/+ ), does not. Two-way ANOVA indicates significant differences due to genotype $\left(F_{(4,75)}=5.824, p=0.0004\right)$, RU feeding $\left(F_{(1,75)}=57.11, p<0.0001\right)$, and interaction between genotype and feeding $\left(F_{(4,75)}=9.168, p<0.0001\right)$. ${ }^{* * *} p<0.0001$ and ${ }^{*} p<0.05$ compared with RU - controls, as determined by Bonferroni post hoc tests. 


\section{References}

Alberini CM (2009) Transcription factors in long-term memory and synaptic plasticity. Physiol Rev 89:121-145. CrossRef Medline

Awasaki T, Lee T (2011) New tools for the analysis of glial cell biology in Drosophila. Glia 59:1377-1386. CrossRef Medline

Awasaki T, Lai SL, Ito K, Lee T (2008) Organization and postembryonic development of glial cells in the adult central brain of Drosophila. J Neurosci 28:13742-13753. CrossRef Medline

Brand AH, Perrimon N (1993) Targeted gene expression as a means of altering cell fates and generating dominant phenotypes. Development 118: 401-415. Medline

Buffington SA, Huang W, Costa-Mattioli M (2014) Translational control in synaptic plasticity and cognitive dysfunction. Annu Rev Neurosci 37:1738. CrossRef Medline

Butler SJ, Ray S, Hiromi Y (1997) klingon, a novel member of the Drosophila immunoglobulin superfamily, is required for the development of the R7 photoreceptor neuron. Development 124:781-792. Medline

Chen CC, Wu JK, Lin HW, Pai TP, Fu TF, Wu CL, Tully T, Chiang AS (2012) Visualizing long-term memory formation in two neurons of the Drosophila brain. Science 335:678-685. CrossRef Medline

Comas D, Petit F, Preat T (2004) Drosophila long-term memory formation involves regulation of cathepsin activity. Nature 430:460-463. CrossRef Medline

Costa RM, Drew C, Silva AJ (2005) Notch to remember. Trends Neurosci 28:429-435. CrossRef Medline

Crittenden JR, Skoulakis EM, Han KA, Kalderon D, Davis RL (1998) Tripartite mushroom body architecture revealed by antigenic markers. Learn Mem 5:38-51. Medline

Doherty J, Logan MA, Taşdemir OE, Freeman MR (2009) Ensheathing glia function as phagocytes in the adult Drosophila brain. J Neurosci 29:47684781. CrossRef Medline

Dura JM, Preat T, Tully T (1993) Identification of linotte, a new gene affecting learning and memory in Drosophila melanogaster. J Neurogenet 9:114. CrossRef Medline

Halter DA, Urban J, Rickert C, Ner SS, Ito K, Travers AA, Technau GM (1995) The homeobox gene repo is required for the differentiation and maintenance of glia function in the embryonic nervous system of Drosophila melanogaster. Development 121:317-332. Medline

Hyden H, Egyhazi E (1962) Nuclear RNA changes of nerve cells during a learning experiment in rats. Proc Natl Acad Sci U S A 48:1366-1373. CrossRef Medline

Hydén H, Lange PW (1970) S100 brain protein: correlation with behavior. Proc Natl Acad Sci U S A 67:1959-1966. CrossRef Medline

Isabel G, Pascual A, Preat T (2004) Exclusive consolidated memory phases in Drosophila. Science 304:1024-1027. CrossRef Medline

Kaltschmidt B, Ndiaye D, Korte M, Pothion S, Arbibe L, Prüllage M, Pfeiffer J, Lindecke A, Staiger V, Israel A, Kaltschmidt C, Mémet S (2006) NFkappaB regulates spatial memory formation and synaptic plasticity through protein kinase A/CREB signaling. Mol Cell Biol 26:2936-2946. CrossRef Medline

Kerr KS, Fuentes-Medel Y, Brewer C, Barria R, Ashley J, Abruzzi KC, Sheehan A, Tasdemir-Yilmaz OE, Freeman MR, Budnik V (2014) Glial wingless/ Wnt regulates glutamate receptor clustering and synaptic physiology at the Drosophila neuromuscular junction. J Neurosci 34:2910-2920. CrossRef Medline

Lee BP, Jones BW (2005) Transcriptional regulation of the Drosophila glial gene repo. Mech Dev 122:849-862. CrossRef Medline

MacDonald JM, Beach MG, Porpiglia E, Sheehan AE, Watts RJ, Freeman MR (2006) The Drosophila cell corpse engulfment receptor Draper mediates glial clearance of severed axons. Neuron 50:869-881. CrossRef Medline

Matsuno M, Horiuchi J, Tully T, Saitoe M (2009) The Drosophila cell adhesion molecule klingon is required for long-term memory formation and is regulated by Notch. Proc Natl Acad Sci U S A 106:310-315. CrossRef Medline

Miyashita T, Oda Y, Horiuchi J, Yin JC, Morimoto T, Saitoe M (2012) $\mathrm{Mg}(2+)$ block of Drosophila NMDA receptors is required for long-term memory formation and CREB-dependent gene expression. Neuron 74: 887-898. CrossRef Medline

Muthukumar AK, Stork T, Freeman MR (2014) Activity-dependent regulation of astrocyte GAT levels during synaptogenesis. Nat Neurosci 17: 1340-1350. CrossRef Medline

Nicholson L, Singh GK, Osterwalder T, Roman GW, Davis RL, Keshishian H (2008) Spatial and temporal control of gene expression in Drosophila using the inducible GeneSwitch GAL4 system. I. Screen for larval nervous system drivers. Genetics 178:215-234. CrossRef Medline

Pannasch U, Rouach N (2013) Emerging role for astroglial networks in information processing: from synapse to behavior. Trends Neurosci 36: 405-417. CrossRef Medline

Pláteník J, Kuramoto N, Yoneda Y (2000) Molecular mechanisms associated with long-term consolidation of the NMDA signals. Life Sci 67:335364. CrossRef Medline

Skoulakis EM, Davis RL (1996) Olfactory learning deficits in mutants for leonardo, a Drosophila gene encoding a 14-3-3 protein. Neuron 17:931944. CrossRef Medline

Steward O, Torre ER, Tomasulo R, Lothman E (1991) Neuronal activity up-regulates astroglial gene expression. Proc Natl Acad Sci U S A 88: 6819-6823. CrossRef Medline

Su H, O'Dowd DK (2003) Fast synaptic currents in Drosophila mushroom body Kenyon cells are mediated by alpha-bungarotoxin-sensitive nicotinic acetylcholine receptors and picrotoxin-sensitive GABA receptors. J Neurosci 23:9246-9253. Medline

Suzuki A, Stern SA, Bozdagi O, Huntley GW, Walker RH, Magistretti PJ, Alberini CM (2011) Astrocyte-neuron lactate transport is required for long-term memory formation. Cell 144:810-823. CrossRef Medline

Tully T, Preat T, Boynton SC, Del Vecchio M (1994) Genetic dissection of consolidated memory in Drosophila. Cell 79:35-47. CrossRef Medline

Xiong WC, Okano H, Patel NH, Blendy JA, Montell C (1994) repo encodes a glial-specific homeo domain protein required in the Drosophila nervous system. Genes Dev 8:981-994. CrossRef Medline

Yuasa Y, Okabe M, Yoshikawa S, Tabuchi K, Xiong WC, Hiromi Y, Okano H (2003) Drosophila homeodomain protein REPO controls glial differentiation by cooperating with ETS and BTB transcription factors. Development 130:2419-2428. CrossRef Medline

Zwarts L, Van Eijs F, Callaerts P (2014) Glia in Drosophila behavior. J Comp Physiol A:1-15. doi: 10.1007/s00359-014-0952-9. 